Research Article

\title{
Novel Evaporation Process for Deposition of Kesterite Thin Films Synthesized by Solvothermal Method
}

\author{
J. A. Estrada-Ayub, L. Álvarez Contreras, M. Román Aguirre, J. G. Murillo Ramírez, \\ M. T. Ochoa-Lara, P. Pizá-Ruiz, and A. Aguilar Elguezabal
}

Centro de Investigación en Materiales Avanzados, S.C., Miguel de Cervantes 120, Complejo Industrial Chihuahua, 31136 Chihuahua, CHIH, Mexico

Correspondence should be addressed to A. Aguilar Elguezabal; alfredo.aguilar@cimav.edu.mx

Received 30 June 2017; Revised 5 October 2017; Accepted 16 October 2017; Published 17 December 2017

Academic Editor: Germà Garcia-Belmonte

Copyright $\odot 2017$ J. A. Estrada-Ayub et al. This is an open access article distributed under the Creative Commons Attribution License, which permits unrestricted use, distribution, and reproduction in any medium, provided the original work is properly cited.

Kesterite, a quaternary compound of $\mathrm{Cu}_{2} \mathrm{ZnSnS}_{4}$, is a promising option as a material absorber to reduce the cost of photovoltaic solar cells. The solvothermal method is a way to synthesize nanoparticles of this material. In this work, once synthesized, particles were deposited on a substrate through evaporation, and their morphological, structural, and optical properties were studied. Results show that changes of precursor ratios during solvothermal synthesis result in a modification of particle morphology but not on its size. The deposition of already synthesized kesterite through evaporation preserves kesterite structure and permits the formation of a homogenous film on a substrate. Optical reflectance and transmittance measurements allowed estimating the band-gap energy between 1.41 and $1.46 \mathrm{eV}$ for representative samples, which is near the optimum for the absorber material.

\section{Introduction}

Thin-film solar cells have emerged recently as a promising option to reduce costs in comparison to current solar photovoltaic technologies. The thin-film solar cells are composed of material layers where each different material layer has a particular function in the working cell. A simplified classification of these layers is as follows: the first is a contact layer deposited over the substrate; the second, over the contact layer, is an energy absorber material; and the third, on the absorber layer, serves as a buffer. Finally, the last is a window layer made of two different materials, acting as a transparent electrode [1]. Among all these material layers, the absorber material is the one that transforms photons into energy. Therefore, absorber materials largely define thin-film solar cells. Some absorbers have been extensively developed and have reached commercial scale, including amorphous silicon, cadmium telluride (CdTe), copper indium selenide (CIS), and copper indium gallium selenide (CIGS). However, these materials have some drawbacks; for example, amorphous silicon requires a lot of energy to be manufactured. Cadmium, on the other hand, is a heavy metal, which may cause environmental and health problems if it is used massively in the production of solar cells. Finally, CIGS cells are composed of elements that are relatively scarce in the earth crust. Therefore, the cost of materials may become a problem in the massive development of affordable photovoltaic cells. Because of these problems, there is an ongoing research to develop substitute options. Among all these options, kesterite has emerged as a promising substitute for CIGS. Kesterite is a quaternary compound material formed by copper, zinc, tin, and sulphur, with the acronym CZTS. Kesterite was first reported by Ito and Nakazawa in 1988 [2]. Research on methods to prepare kesterite thin films can be classified into three types: (1) Research whose purpose is to synthesize materials, for example, nanoparticles [3-5]. (2) Research about deposition of material once it is synthesized. Some of these deposition methods already reported are roll to roll process [6] and spray coating [7]. (3) Finally, research about methods 
used for simultaneous synthesis and deposition of several materials over the substrate [8-10]. In the present work are reported CZTS compounds synthesized by using the solvothermal method and deposited over a substrate by thermal evaporation. Usually, thermal evaporation is applied using elemental metals in a sulphur atmosphere [11, 12]. To the best of our knowledge, the present paper reports for the first time a CZTS compound applied directly through thermal evaporation without a sulfurized vapor filling the atmosphere.

\section{Materials and Methods}

2.1. CZTS Solvothermal Methods. CZTS was synthesized following the experimental procedure reported by Zhou et al. [13]. The procedure consists in the use of ethylene alcohol, copper (II) chloride dihydrate $\left(\mathrm{CuCl}_{2} \cdot 2 \mathrm{H}_{2} \mathrm{O}\right)$, zinc (II) chloride $\left(\mathrm{ZnCl}_{2}\right)$, tin (IV) chloride tetrahydrate $\left(\mathrm{SnCl}_{4} \cdot 4 \mathrm{H}_{2} \mathrm{O}\right)$, and thiourea $\left(\mathrm{CH}_{4} \mathrm{~N}_{2} \mathrm{~S}\right)$ which were all of analytical grade from Sigma-Aldrich, and Polyvinylpyrrolidone (PVP) was a product of Sigma. The experimental procedure included $2 \mathrm{mmol}$ copper (II) chloride dihydrate, $1 \mathrm{mmol}$ zinc (II) chloride, $1 \mathrm{mmol}$ tin (IV) chloride tetrahydrate, and $5 \mathrm{mmol}$ thiourea. Three different samples were prepared with polyvinylpyrrolidone (PVP): (1) one of these samples with $0.640 \mathrm{~g}$ of PVP, (2) a second sample with $0.324 \mathrm{~g}$, and (3) a third sample with $0.964 \mathrm{~g}$ of PVP. All three samples were prepared with $38 \mathrm{ml}$ of ethylene alcohol and loaded into a $40 \mathrm{ml}$ Teflon-lined stainless autoclave and kept in an oven at $200^{\circ} \mathrm{C}$ for 24 hours. The resultant materials were washed several times with distilled water and centrifuged, and the materials washed with water were washed again with absolute ethanol and centrifuged.

The resultant materials were characterized and applied to an Mo-glass and Corning glass square substrates of $25 \times 25 \mathrm{~mm}$. These materials were deposited on the substrates via a Cressington Carbon Coater. In fact, this method was modified to improve the deposition process on the substrates. In order to control the quantity of the deposited material onto substrates were varied both the time and the magnitude of the electric current (amperage) applied. In the method used to evaporate the synthesized materials, these materials were first preheated by applying a current of $20 \mathrm{~A}$ to the electrode in the experimental setup. Next was applied an electric current of $21 \mathrm{~A}$ for $10 \mathrm{~s}$. Finally, the current was increased by $1 \mathrm{~A}$ for every $10 \mathrm{~s}$ until it reaches $35 \mathrm{~A}$.

2.2. CZTS Characterization. Morphology and size of the synthesized materials and the deposited films were characterized by scanning electron microscopy (SEM; JEOL JSM7000F) equipped with high resolution energy dispersive X-ray spectroscopy (EDS, EDAX Genesis); particles were dispersed in absolute ethanol using sonication for five minutes and deposited on a silicon wafer. X-ray diffraction (XRD) was made using a PANalytical X Pert Pro X-ray diffractometer, with a scanning rate of $0.1^{\circ} / 2.5 \mathrm{~s}$, using $\mathrm{CuK}_{\alpha}$ radiation and a wavelength $\lambda$ of $1.540 \mathrm{~nm}$. In addition, selected samples were characterized by high-resolution transmission electron microscopy (HRTEM; Jeol JEM 2200FS), equipped with spherical aberration correction at an operation voltage of $200 \mathrm{kV}$. On the other hand, thin films were also characterized by using an FIB equipment (Focus Ion Beam, FEI Quanta 3D FEG Dual Beam, operation voltage $30 \mathrm{kV}$ ). By using UV-Vis-NIR spectroscopy were measured the optical transmission and optical reflectance in the UV-Vis-NIR ranges employing an Agilent Cary 5000 spectrophotometer. The optical transmittance $T$ was measured at normal incidence, and the optical reflectance $R$ was obtained using an absolute specular reflectance accessory with an incident light beam at $7^{\circ}$ to the sample surface. From these measurements, the optical absorption coefficient $\alpha$ in the UV-Vis-NIR ranges of the electromagnetic spectrum of each one of the samples was obtained. Then, the direct band gap for each one of the samples was evaluated from the absorption coefficient. The samples were characterized by Raman spectroscopy using an excitation wavelength $632.8 \mathrm{~nm}$ emitted by a laser power of $9 \mathrm{MW}$ in a Horiba model Labram HR Vis633 spectrometer. In order to avoid structural changes due to the thermal effect, the Raman measurements were made at lower power density.

\section{Results and Discussion}

3.1. Morphology Characterization. Figure 1 shows SEM images of the morphology and size of prepared particles. As it can be seen from Figures $1(\mathrm{a})-1(\mathrm{~g})$, as PVP increases, the morphology of particles changes from needle-flower to spherical shape. This is in accordance with the results reported by [14]. The obtained results in the present research show how particles synthesized reached nanoscale size, but they remain sintered. Therefore, PVP does not control particle size and the sintered characteristic of them, but it does control its morphology. Flower-shaped material shown in Figure 1(a) was also characterized by HRTEM. Figures 2(a)-2(c) show a detail of the flower-like morphology of the particle shown in Figure 1(a).

From the EDS analysis of the synthesized materials, it was observed a $20 \%$ reduction of copper, regarding the stoichiometry values, a $16 \%$ reduction of zinc, and a $11 \%$ increase of sulphur, while tin remains close to its stoichiometry value. In order to verify the elements present in the sample synthesized with $0.964 \mathrm{~g}$ of PVP, shown in Figure 1(g), additionally, it was characterized by means of a particle elemental analysis. The results concerning the composition of this sample are shown in Figure 3. In this last figure, it can be appreciated that all the elements from the quaternary compound, copper, zinc, tin, and sulphur are present in the correct proportion in the particle. Therefore, these results support the identification of this particle as kesterite. Figures $4(\mathrm{~b})-4(\mathrm{e})$ show the spatial distribution of constituent elements $\mathrm{Cu}, \mathrm{Zn}, \mathrm{Sn}$, and $\mathrm{S}$, respectively, corresponding to sample (a) synthesized with $0.964 \mathrm{~g}$ of PVP. Figure 5 shows a scheme describing the effect of PVP on particle formation during the synthesis of CZTS. The precursors $\mathrm{CuCl}_{2}, \mathrm{ZnCl}_{2}$, and $\mathrm{SnCl}_{2}$ provide copper, zinc, and tin, respectively, while thiourea is the source of sulphur. All these components were kept constant, while the quantity of PVP was varied from $0.234 \mathrm{~g}$ to $0.964 \mathrm{~g}$. The results show changes in the particle morphology from flower like to sphere like. However, in order to verify the crystalline structure and their differences, an XRD analysis was completed, and the 


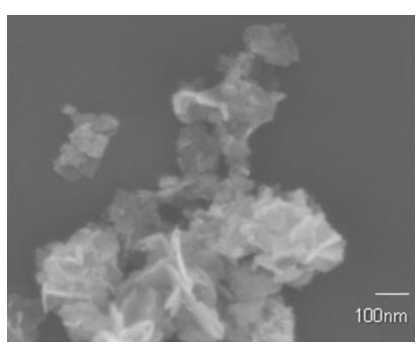

(a)

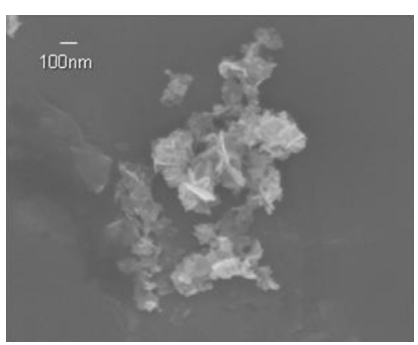

(e)

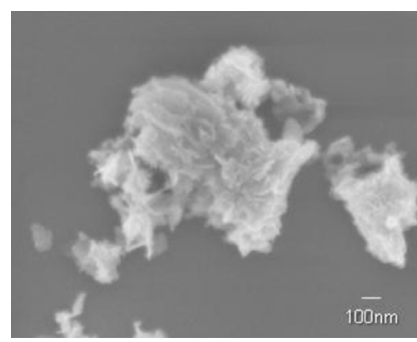

(b)

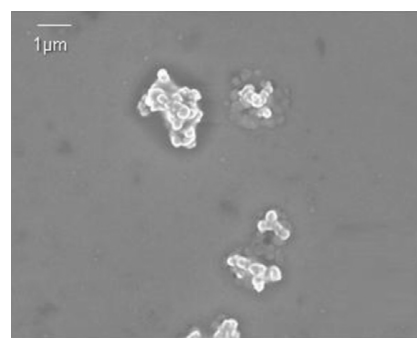

(f)

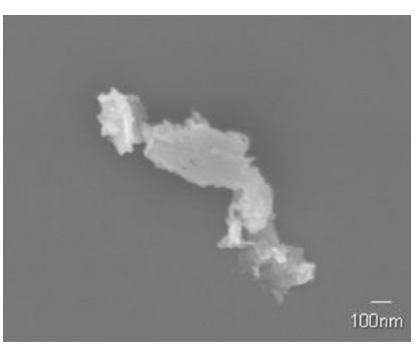

(c)

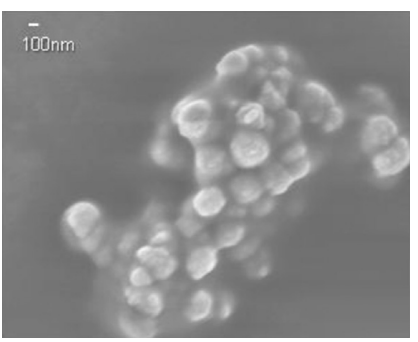

(g)

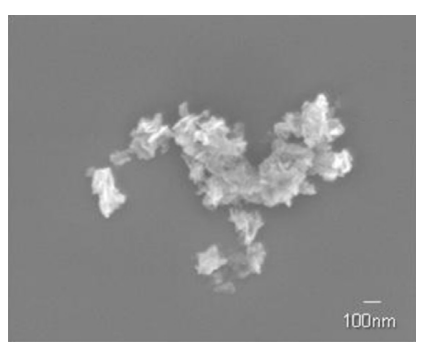

(d)

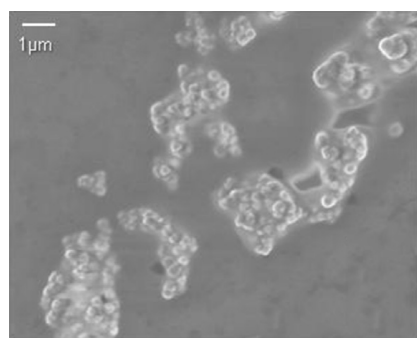

(h)

FiguRE 1: SEM images of morphology and sizes of prepared particles, (a) and (e) correspond to samples with $0.324 \mathrm{~g}$ of PVP; (b), (c), and (d) correspond to samples with $0.640 \mathrm{~g}$ of PVP, and (f), (g), and (h) correspond to samples with $0.964 \mathrm{~g}$ of PVP.

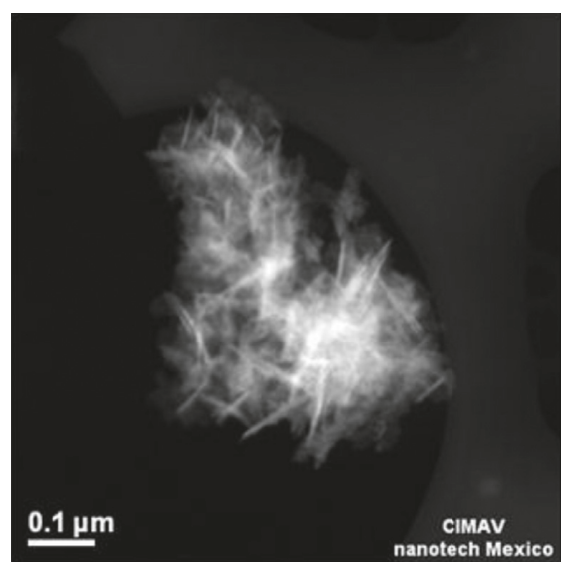

(a)

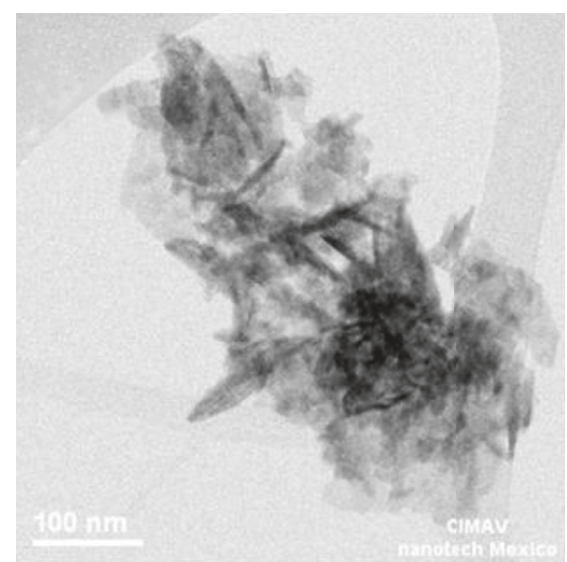

(b)

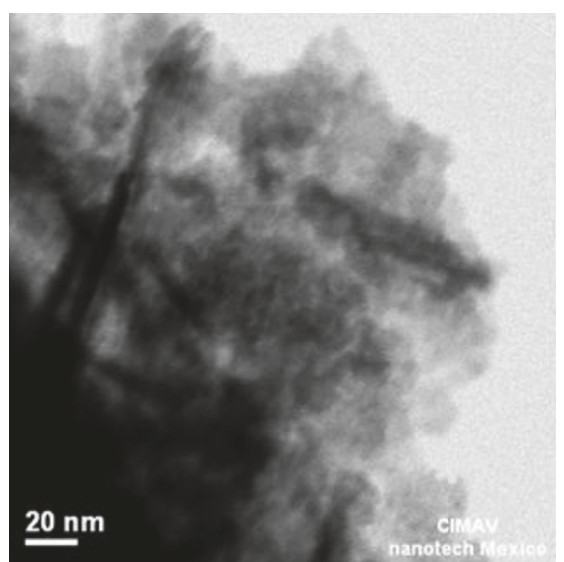

(c)

FIgURE 2: HRTEM images showing materials synthesized using $0.324 \mathrm{~g}$ of PVP. These different three particles shown at (a), (b), and (c) support the assumption that the morphology is a general characteristic of all the materials and not only a particular defect.

crystallite size of the samples was determined by the Scherrer equation, being required the deconvolution of peaks to determine the full width at half maximum (FWHD). The samples were also characterized by EDS analysis as powders once the materials were evaporated over the substrate. The results are shown in Table 1.

3.2. XRD Characterization. The results of the X-ray diffraction study, shown in Figure 6, allowed identifying the crystallographic structure of the samples synthesized with different amounts of PVP $(0.3324 \mathrm{~g}, 0.640 \mathrm{~g}$, and $0.964 \mathrm{~g})$. In this figure, it can be appreciated that the peaks of the three diffraction patterns coincide exactly with those of the pure phase of kesterite structure (CZTS) (JCPDS 01-75-4122).
No peaks of impurities were detected. In other words, the structure of CZTS is the same for all samples, despite having different percentages of PVP and morphologies. In Figure 6, it can be seen how the main diffraction peaks are present in all samples, supporting the assumption that despite any changes in PVP, kesterite is formed. Based on diffraction data, the crystallite size was calculated according to the Scherrer formula, and the necessary information such as FWHD and the degrees was solved through deconvolution of the peaks using Gaussian curves.

3.3. Evaporation of Materials Over a Substrate. Synthesized materials were deposited over a Corning glass and an Mocoated glass. Figure 7 shows an assembly of CZTS deposited 
Comment: 3CZZTSHT 190614 PVP 964

\begin{tabular}{ccc}
\hline Element & Weight \% & Atomic \% \\
\hline C K & 12.05 & 31.73 \\
O K & 3.66 & 7.23 \\
Si K & 22.16 & 24.95 \\
S K & 17.98 & 17.73 \\
C1 K & 0.90 & 0.80 \\
Cu L & 17.40 & 8.66 \\
Zn L & 9.25 & 4.48 \\
Sn L & 16.60 & 4.42 \\
Totals & 100.00 & \\
\hline
\end{tabular}

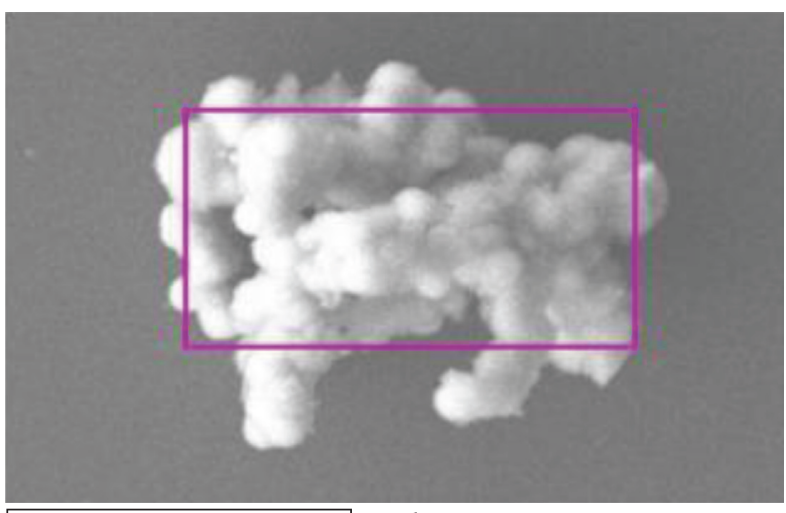

$10 \mu \mathrm{m} \quad$ Electron image 1

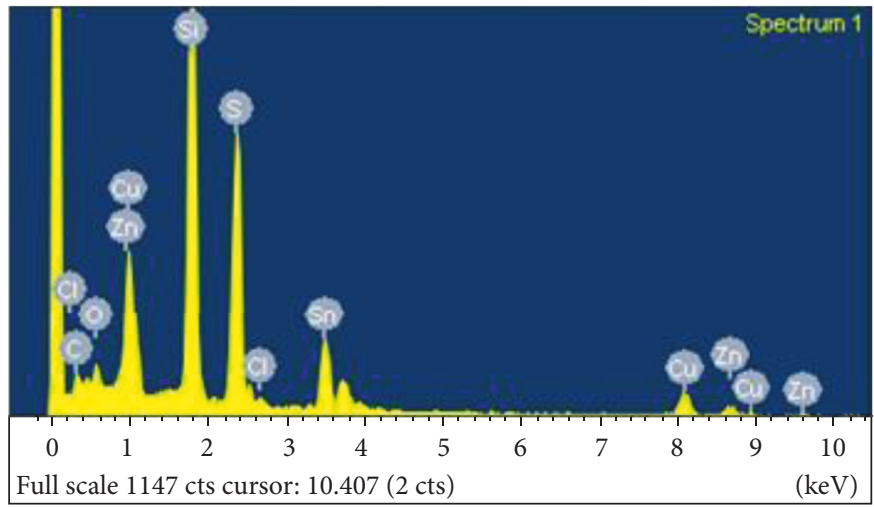

FIgURE 3: Composition of a representative sample, synthesized with $0.964 \mathrm{~g}$ of PVP, obtained from EDS analysis.

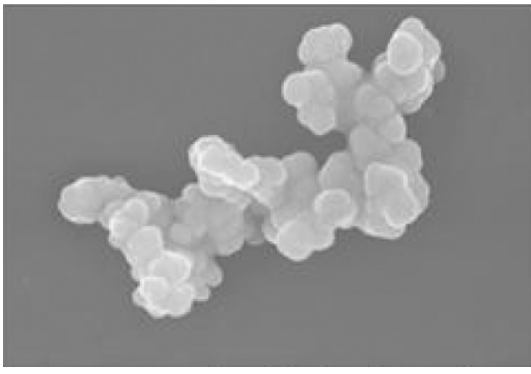

$3 \mu \mathrm{m}$

Electron image 1 (a)

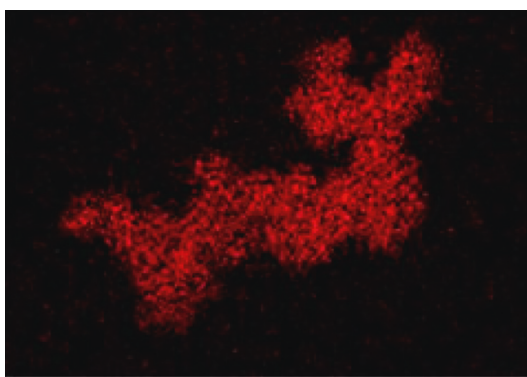

S Kal

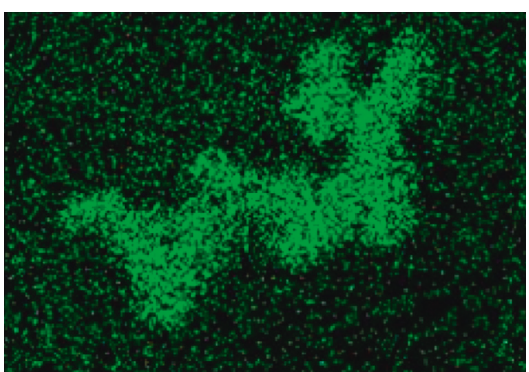

Cu La1_2

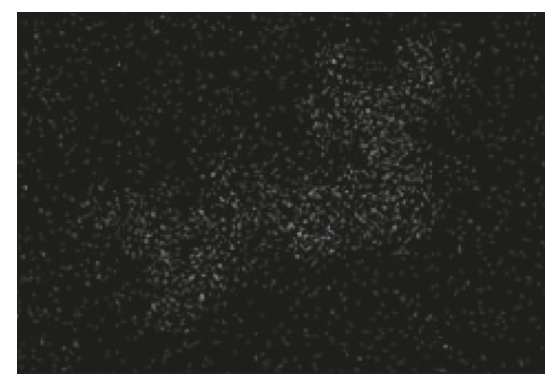

Sn Lal

(c)

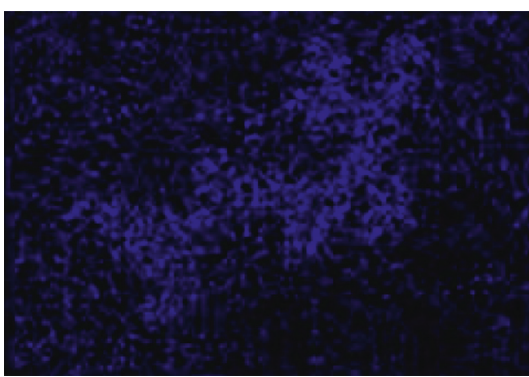

Zn Lal_2

(d)

(e)

Figure 4: Particle elemental analysis of a sample synthesized with $0.964 \mathrm{~g}$ of PVP that shows that the principal elements of kesterite are found in the entire particle. (a) Morphology of the sample. EDS map of (b) copper, (c) tin, (d) sulphur, and (e) zinc. 
EFFECT OF PVP ON PARTICLES FORMATION

CZTS particles formation as PVP (Polyvinylpyrrolidone) increases, shape changes from flower like to sphere.
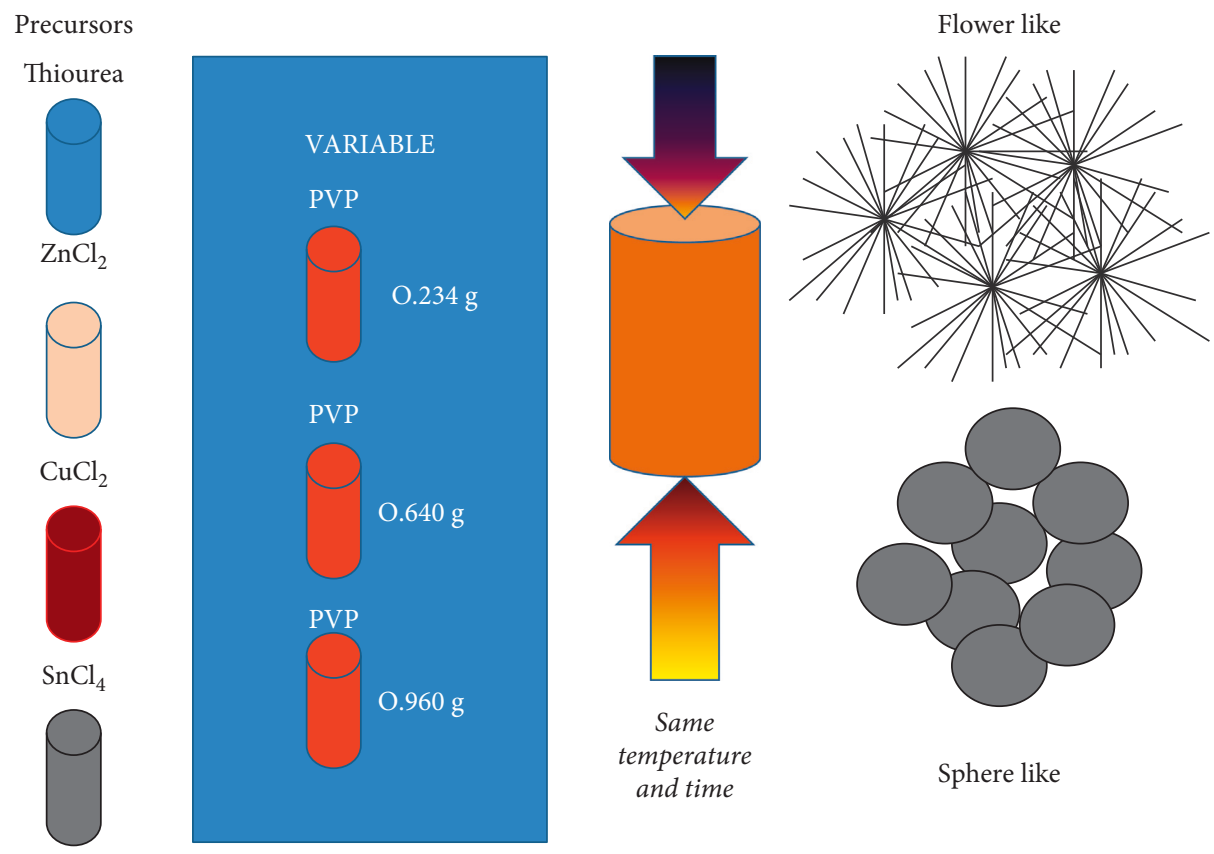

FIGURE 5: Scheme describing the effect of PVP on particle formation during the synthesis process of CZTS from left to right: precursors are shown; then in the box, the PVP as a variable introduced in the experiment; the process; and the different results in morphology.

TABLE 1: XRD diffraction study and EDS analysis of the CZTS synthesized with different PVP contents.

\begin{tabular}{lcccccccccccccc}
\hline Sample & \multicolumn{4}{c}{ X-ray diffraction crystallite size $(\mathrm{nm})$} & \multicolumn{4}{c}{ Electron dispersion spectroscopy (atomic \%) } \\
CZTS/PVP & \multicolumn{3}{c}{ Crystallographic planes } & Average & $\mathrm{Cu}$ & $\mathrm{Cu}$ & Zn & Zn & Sn & Sn & S & S \\
& $(112)$ & $(004)$ & $(220)$ & $(312)$ & crystallite size & bulk & on film & bulk & on film & bulk & on film & bulk & on film \\
\hline CZTS/0.324g & 9.88 & 10.48 & 12.71 & 10.97 & 11.0 & 24.62 & 4.91 & 3.16 & 1.97 & 8.29 & 29.00 & 33.20 & 64.12 \\
CZTS/0.640 g & 10.05 & 25.16 & 13.23 & 10.14 & 14.6 & 27.47 & 8.66 & 5.60 & 8.84 & 10.28 & 23.21 & 37.83 & 59.29 \\
CZTS/0.964g & 8.72 & 8.72 & 11.63 & 11.95 & 10.2 & 13.00 & 11.61 & 3.00 & 7.22 & 24.00 & 44.6 & 55.00 & 36.66 \\
\hline
\end{tabular}

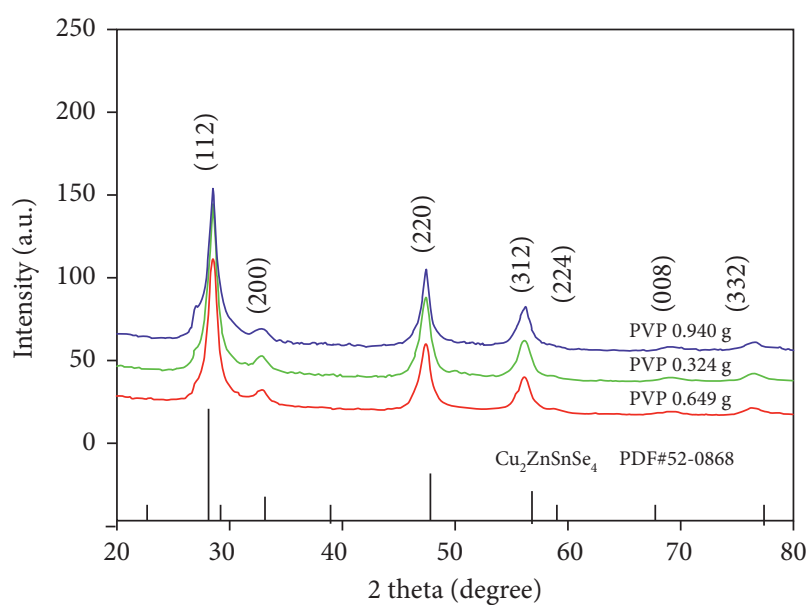

FIGURE 6: XRD patterns of CZTS samples prepared with different concentrations of PVP which show a good matching between the peaks in each one of the diffraction patterns and those of the pure phase of kesterite structure (CZTS) (JCPDS 01-75-4122).

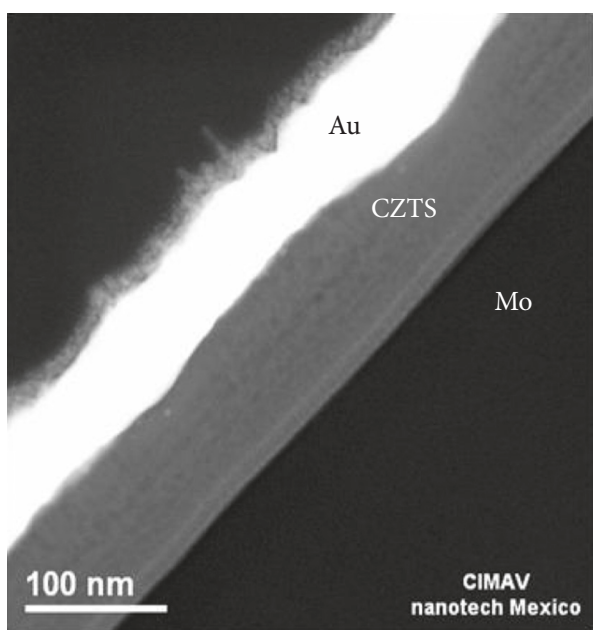

FIgURE 7: HRTEM image of the cross section of an assembly of CZTS and Mo. The image shows the advantages of the physical vapor deposition process on producing extremely homogeneous thin films. 
over a glass substrate. The sample chosen to make the assembly was that containing the particles with the smaller size, which were prepared with $0.324 \mathrm{~g}$ of PVP. By using the method of evaporation, it was possible to deposit thin films with a thickness of the order of $50 \mathrm{~nm}$. In a typical assembly obtained in this work, shown in Figure 7, molybdenum was deposited through a sputtering process, and CZTS were deposited through evaporation. Thin films deposited over the glass substrate were prepared using an focused ion beam (FIB) in order to see the cross section using HRTEM. In Figure 7, it can be seen that the deposited material is homogeneous. In fact, the different layers of the same material were deposited in different stages varying the amperage and deposition time as it was previously discussed in Section 2.1. Three different powders synthesized with different PVP contents were deposited through physical evaporation over a glass substrate and characterized through SEM and EDS, and the results are shown in Table 1. XRD results were compared with the EDS data for each of the synthesized material as well as the same material deposited through evaporation method. Results indicate that there are small changes in crystallite size according to the PVP content, but there are major changes in the stoichiometric ratio of the elements when they undergo a physical evaporation phase. The most important changes are the increase in the $\mathrm{Zn}$ content; there is also an increase in the sulphur content and a decrease in the $\mathrm{Cu}$ content when they undergo this physical change through evaporation.

3.4. UV-Vis-NIR Spectroscopy. UV-Vis spectroscopy studies included measurements of optical transmittance and optical reflectance in the wavelength ranging from 200 to $2300 \mathrm{~nm}$ as shown in Figure 8, for the CZTS assembly synthetized with $0.324 \mathrm{~g}$ of PVP and deposited through thermal evaporation on a glass substrate. As can be seen in Figure 8, one of the representative assemblies of CZTS studied in this work is highly transparent above a wavelength of $350 \mathrm{~nm}$. Next, these measurements of transmittance and reflectance were used to obtain the absorption coefficient $\alpha$ which was used to calculate the direct band gap of CZTS assembly using the relation: $(\alpha h \nu)^{2}=A\left(E_{\mathrm{g}}-h \nu\right)$, where $v$ is the frequency of the incident radiation, $h$ is Plank's constant, $E_{\mathrm{g}}$ represents the optical band gap, and $A$ is a constant [15]. Figure 9 shows the behaviour of $(\alpha h \nu)^{2}$ as a function of $h v$ for the sample of CZTS with $0.324 \mathrm{~g}$ of PVP deposited through thermal evaporation on glass. The $E_{\mathrm{g}}$ value was evaluated by extrapolating the linear part of the plots (see solid line) to the abscissa $(h v)$. In the case of CZTS assembly synthetized with $0.324 \mathrm{~g}$ of PVP, the values of $E_{\mathrm{g}}$ calculated was equal to $1.46 \mathrm{eV}$. In fact, the values of $E_{\mathrm{g}}$ of all the CZTS assemblies synthetized in this work were found in the range between 1.41 and $1.46 \mathrm{eV}$, which is very close to the optimum for solar cell assemblies. The maximum experimental error found in the calculation of the band-gap energy was estimated in $0.01 \mathrm{eV}$. These results open the possibility that CZTS samples synthesized in this work can be used as an efficient material in solar cell assemblies.

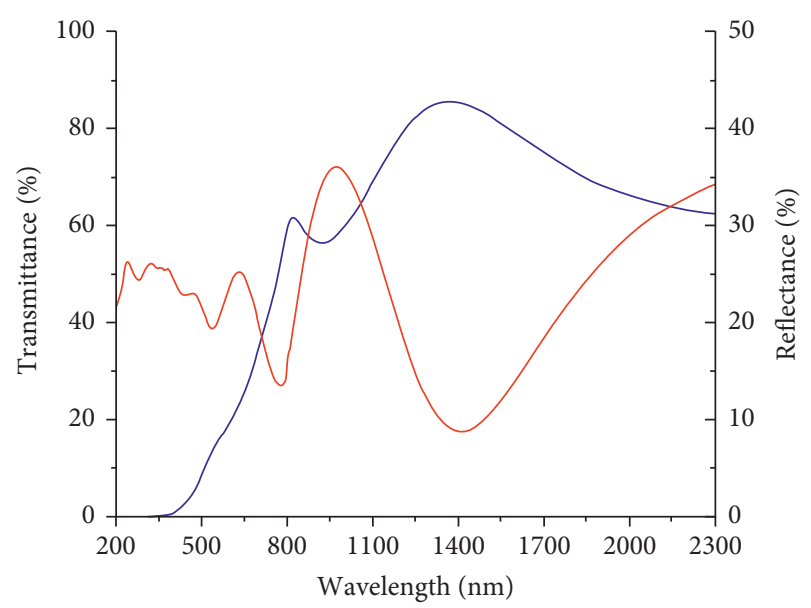

FIgURE 8: Optical transmittance spectra and optical reflectance spectra of CZTS assembly synthetized with $0.324 \mathrm{~g}$ of PVP and deposited through thermal evaporation on glass substrate.

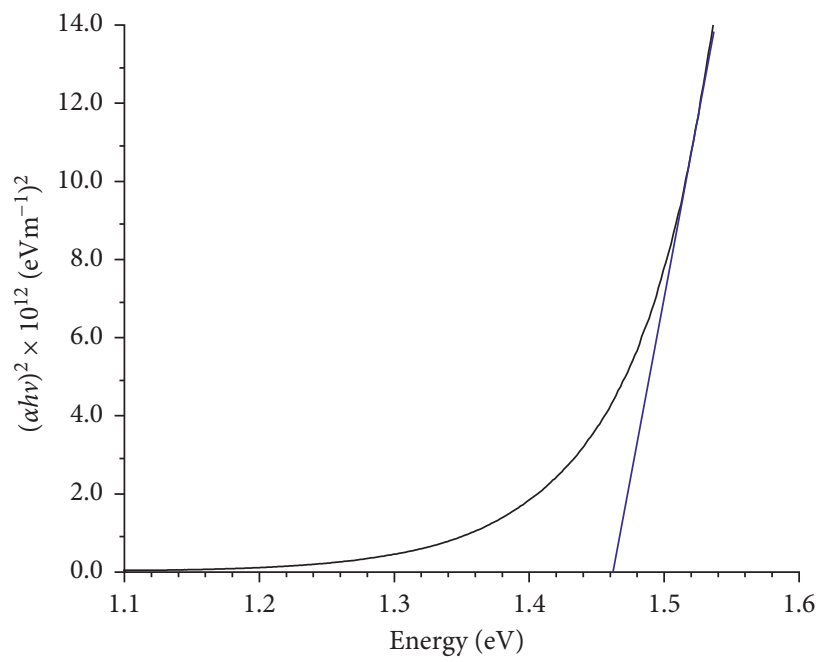

FIGURE 9: $(\alpha h \nu)^{2}$ versus $h v$ spectra for the calculation of direct band gap of CZTS assembly synthetized with $0.324 \mathrm{~g}$ of PVP and deposited by thermal evaporation.

3.5. Raman Scattering Analysis of Kesterite. Figure 10 shows the spectrum measured on three different powders synthesized with different PVP content. From top to bottom, they appear in decreasing order of PVP content, being spectrum marked as (a), the one that was synthesized with the largest PVP content $(0.964 \mathrm{~g})$. Spectrum (b) shows a powder synthesized with $0.640 \mathrm{~g}$ of PVP, and finally spectrum (c) shows a kesterite powder synthesized with $0.324 \mathrm{~g}$ of PVP. The fitting of the spectra with Gaussian curves was also shown in Figure 10 and was allowed to identify two main peaks at $272 \mathrm{~cm}^{-1}$ and at $322 \mathrm{~cm}^{-1}$; the position of these two peaks remain constant in all the three different powders. The position of these two peaks are a good approximation of the signals at $289 \mathrm{~cm}^{-1}$ and $339 \mathrm{~cm}^{-1}$ identified as kesterite peaks [16]. The peak at $272 \mathrm{~cm}^{-1}$ corresponds with the experimental Raman band identified in kesterite with a symmetry $\mathrm{E}(\mathrm{LO})$. The band at $322 \mathrm{~cm}^{-1}$ 


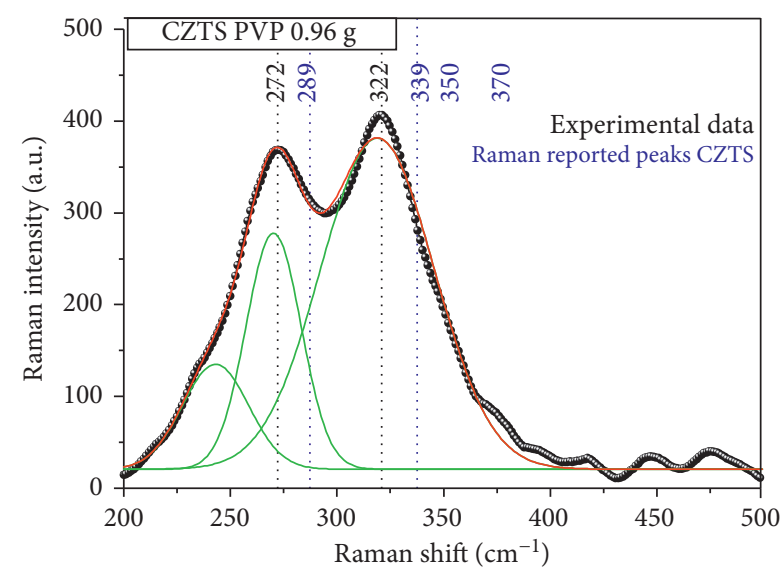

(a)

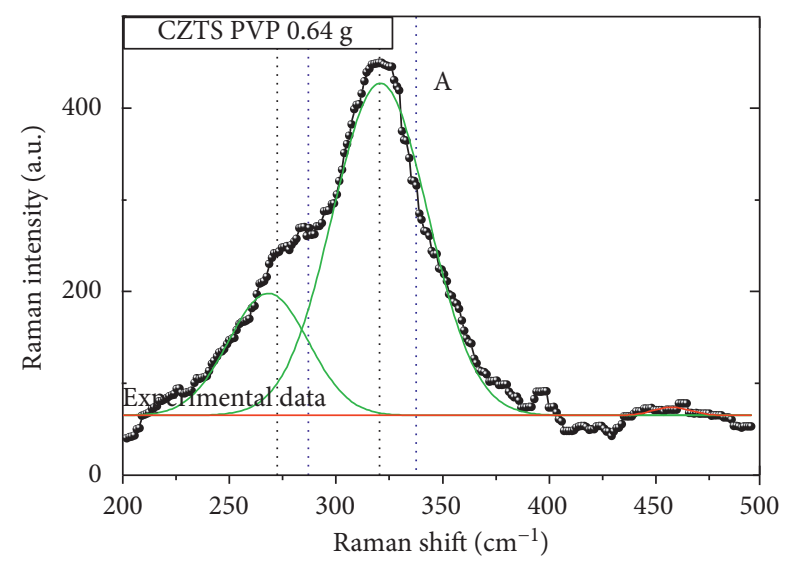

(b)

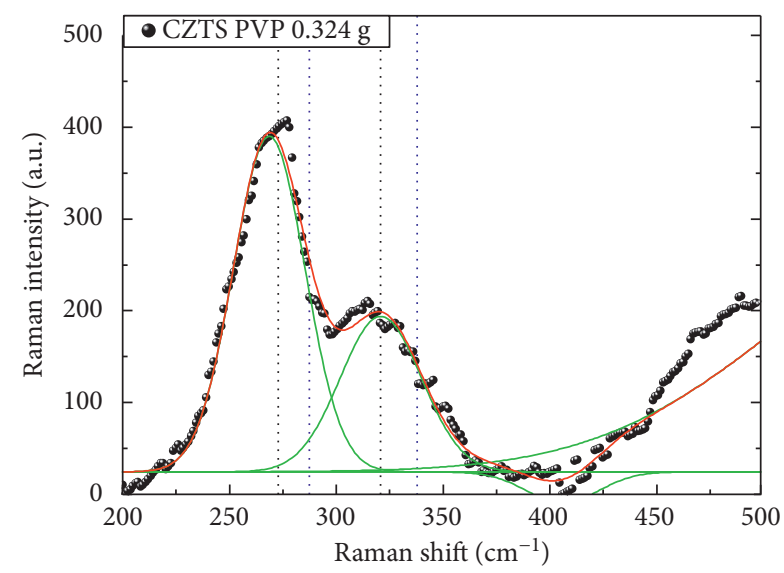

(c)

FIGURE 10: Raman scattering analysis of the CZTS using different PVP contents in their synthesis. Spectra (a) to (c) are ordered from higher to lower content of PVP used during synthesis of the source powders, the PVP content being (a) $0.964 \mathrm{~g}$, (b) $0.640 \mathrm{~g}$, and (c) $0.324 \mathrm{~g}$. Characteristic kesterite Raman peak positions are indicated on top, as well as experimental values resulted from Gaussian analysis.

corresponds with a symmetry $\mathrm{B}(\mathrm{TO})$. B mode is nondegenerated, and $\mathrm{E}$ mode is doubly degenerated. While the $\mathrm{B}$ mode involves in cation displacement along the $C$-direction, $\mathrm{E}$ mode involves in plane cation vibrations parallel to the $a$ direction. The normal displacements of the
24र́ point vibrational mode predict that $\mathrm{B}(\mathrm{TO})$ is produced by a tin and sulphur couple, and the E mode involves a zinc and sulphur [17]. Therefore, the increase in PVP quantity involves a growth in the vibration of the zinc/sulphur and a reduction in copper.

\section{Conclusions}

Kesterite materials were successfully synthesized through a solvothermal method. It was found that the content of PVP allows controlling the particle morphology, but not their size. For the first time, CZTS materials were deposited using thermal metal evaporation controlling its thickness by means of a method based on variations of the electric current intensity and the deposition time. From the HRTEM characterization, it was observed that the deposited materials are very homogeneous. Therefore, the quaternary compound (CZTS) resulting from solvothermal synthesis can be directly deposited onto substrates using the method described in this work. Raman sensibility was key to identify how materials change their structure, according to PVP content. However, these changes do not cause major modifications in the interatomic distances, making it difficult for XRD diffraction, to reflect them. For the first time, a physical evaporation method for CZTS deposition is reported, allowing the control of $\mathrm{Cu} / \mathrm{Zn}$ ratio to obtain an ideal composition for kesterite solar cells. This method will add a new tool to design better photovoltaic devices, since it allows obtaining extreme homogeneous thin films as well as the possibility to deposit materials over flexible substrates. The calculated band gap of CZTS materials deposited through thermal evaporation suggests that this material and the method described in this work can be used for efficient solar cell assemblies.

\section{Conflicts of Interest}

The authors declare that they have no conflicts of interest.

\section{Acknowledgments}

This work was supported in part by CEMIE-Sol (Centro Mexicano de Innovación en Energía Solar). The authors thank NANOTECH (Laboratorio Nacional de Nanotecnología) and their microscope technicians Wilber Antunez and Carlos Elias Ornelas. The authors thank Jose Trinidad Holguin for his support in the development of this work.

\section{References}

[1] B. von Roedern, "How do buffer layers affect solar cell performance and solar cell stability?," MRS Online Proceedings Library Archive, vol. 668, 2001.

[2] K. Ito and T. Nakazawa, "Electrical and optical properties of stannite-type quaternary semiconductor thin films," Japanese Journal of Applied Physics, vol. 27, no. 11, pp. 2094-2097, 1988.

[3] S. A. Vanalakar, G. L. Agwane, M. G. Gang, P. S. Patil, J. H. Kim, and J. Y. Kim, "A mild hydrothermal route to synthesis of CZTS nanoparticle inks for solar cell applications," Physica Status Solidi (c), vol. 12, no. 6, pp. 500-503, 2015. 
[4] L. Qian, J. Yang, R. Zhou et al., "Hybrid polymer-CdSe solar cells with $\mathrm{ZnO}$ nanoparticle buffer layer for improved efficiency and lifetime," Journal of Materials Chemistry, vol. 21, no. 11, pp. 3814-3817, 2011.

[5] Y. Cao, M. S. Denny Jr., J. V. Caspar et al., "High-efficiency solution-processed $\mathrm{Cu}_{2} \mathrm{ZnSn}(\mathrm{S}, \mathrm{Se})_{4}$ thin-film solar cells prepared from binary and ternary nanoparticles," Journal of the American Chemical Society, vol. 134, no. 38, pp. 15644$15647,2012$.

[6] W. Liu, H. Shi, T. R. Andersen et al., "Roll-coating fabrication of ITO-free flexible solar cells based on a non-fullerene small molecule acceptor," RSC Advances, vol. 5, no. 45, pp. 3600136006, 2015.

[7] A. Reale, L. La Notte, L. Salamandra et al., "Spray coating for polymer solar cells: an up-to-date overview," Energy Technology, vol. 3, no. 4, pp. 385-406, 2015.

[8] M. P. Valkonen, S. Lindroos, T. Kanniainen, M. Leskela, U. Tapper, and E. Kauppinen, "Thin multilayer CdS/ZnS films grown by SILAR technique,” Applied Surface Science, vol. 120, no. 1-2, pp. 58-64, 1997.

[9] H. M. Pathan and C. D. Lockhande, "Deposition of metal chalcogenide thin films by successive ionic layer adsorption and reaction (SILAR) method," Bulletin of Materials Science, vol. 27, no. 2, pp. 85-111, 2004.

[10] S. Gupta, T. J. Whittles, Y. Batra et al., "A low-cost, sulfurization free approach to control optical and electronic properties $\mathrm{Cu}_{2} \mathrm{ZnSnS}_{4}$ via precursor variation," Solar Energy Materials and Solar Cells, vol. 157, pp. 820-830, 2016.

[11] L. Zhang, S. Karthikeyan, M. J. Sibakoti, and S. A. Campbell, "Chemical composition dependence of $\mathrm{Cu}_{2} \mathrm{ZnSnS}_{4}$ absorbers fabricated by sulfurization of thermal evaporated precursors and solar cell performance," MRS Online Proceedings Library Archive, vol. 1738, 2015.

[12] B. Shin, O. Gunawan, Y. Zhu, N. A. Bojarczuk, J. S. Chey, and S. Guha, "Thin film solar cell with $8.4 \%$ power conversion efficiency using an earth-abundant $\mathrm{Cu}_{2} \mathrm{ZnSnS}_{4}$ absorber," Progress in Photovoltaics: Research and Applications, vol. 21, no. 1, pp. 72-76, 2013.

[13] Y. L. Zhou, W. H. Zhou, Y. F. Du, M. Li, and S. X. Wu, "Sphere-like kesterite $\mathrm{Cu}_{2} \mathrm{ZnSnS}_{4}$ nanoparticles synthesized by a facile solvothermal method," Materials Letters, vol. 65, no. 11, pp. 1535-1537, 2011.

[14] Y. L. Zhou, W. H. Zhou, M. Li, Y. F. Du, and S. X. Wu, "Hierarchical $\mathrm{Cu}_{2} \mathrm{ZnSnS}_{4}$ particles for a low-cost solar cell: morphology control and growth mechanism," Journal of Physical Chemistry C, vol. 115, no. 40, pp. 19632-19639, 2011.

[15] J. G. Murillo, G. Herrera, A. Vega-Rios, S. Flores-Gallardo, J. Duarte-Moller, and J Castillo-Torres, "Effect of $\mathrm{Zn}$ doping on the photoluminescence properties of $\mathrm{LiNbO}_{3}$ single crystals," Optical Materials, vol. 62, pp. 639-645, 2016.

[16] P. A. Fernandes, P. P. Salome, and A. F. da Cunha, "Study of polycrystalline $\mathrm{Cu}_{2} \mathrm{ZnSnS}_{4}$ films by Raman scattering," Journal of Alloys and Compounds, vol. 509, no. 28, pp. 7600-7606, 2011.

[17] M. Dimitrievska, A. Fairbrother, T. Fontane et al., "Multiwavelength excitation Raman scattering study of polycrystalline kesterite $\mathrm{Cu}_{2} \mathrm{ZnSnS}_{4}$ thin films," Applied Physics Letters, vol. 104, no. 2, p. 021901, 2014. 

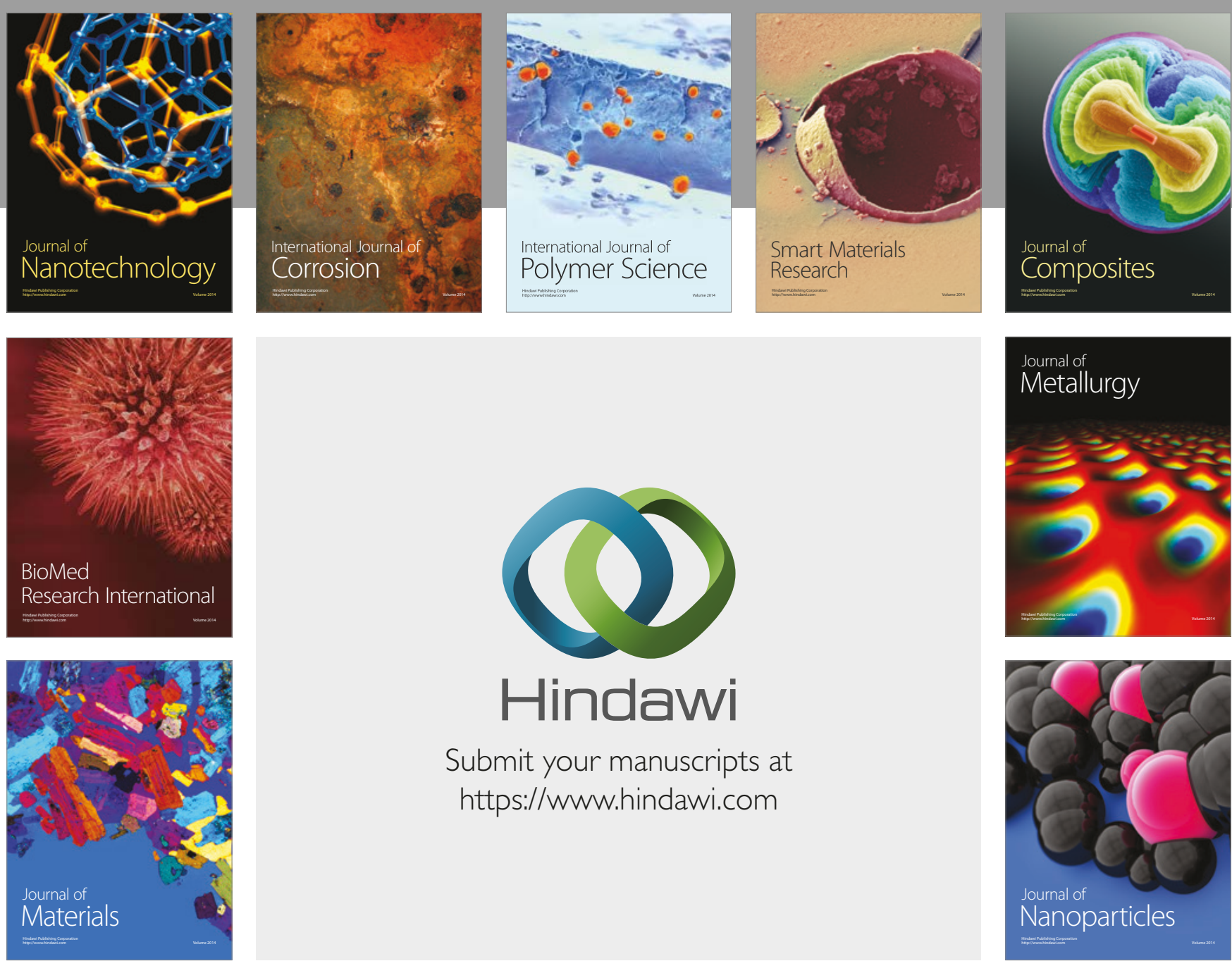

\section{Hindawi}

Submit your manuscripts at

https://www.hindawi.com
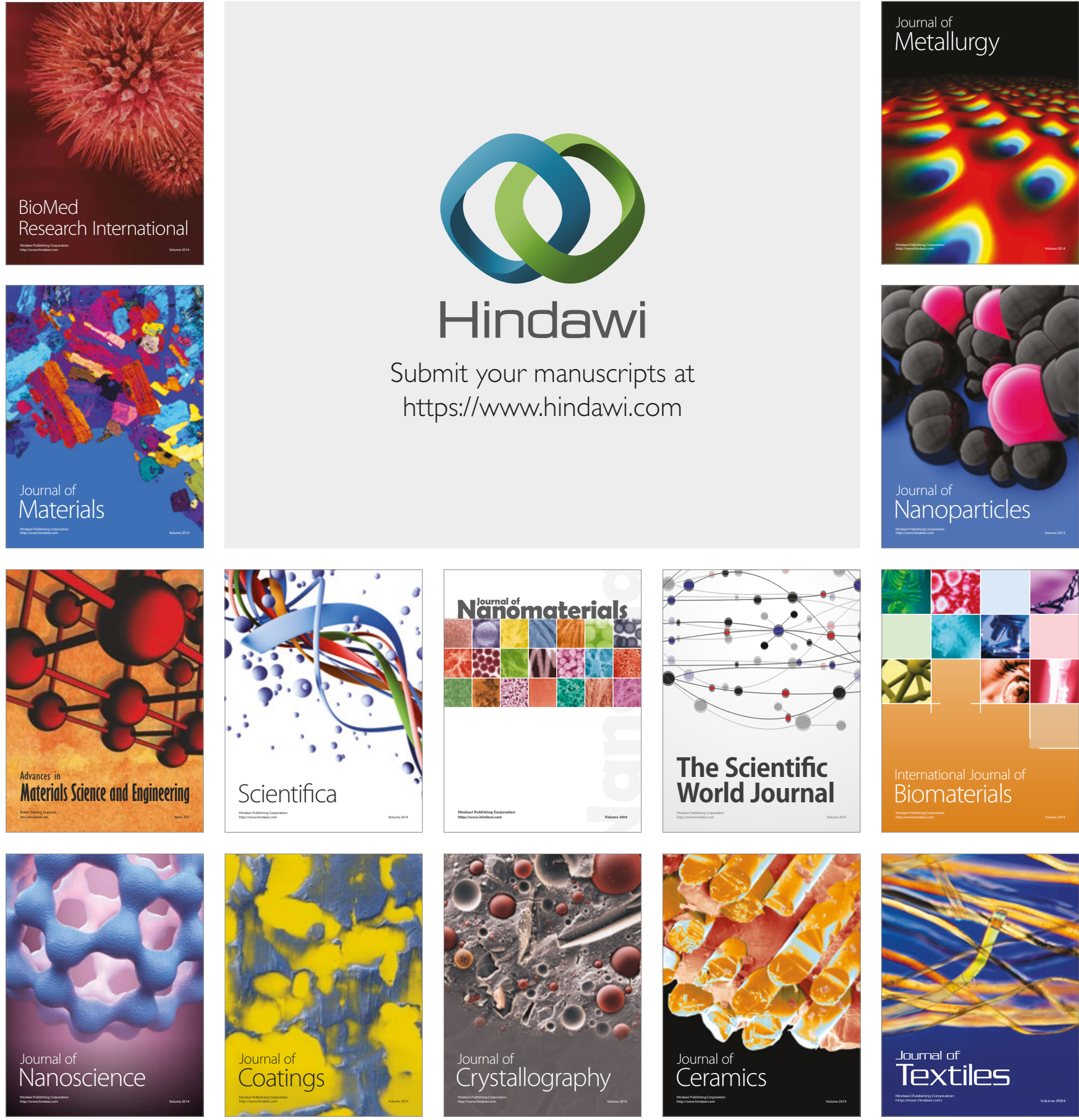

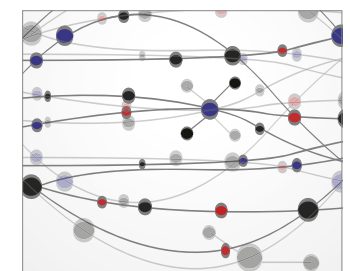

The Scientific World Journal
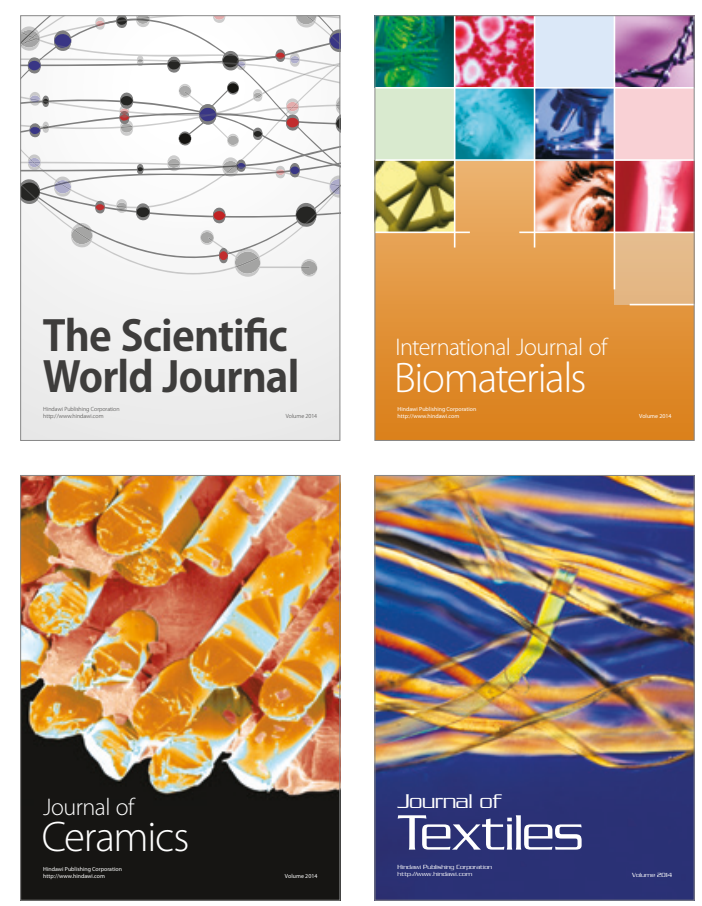